\title{
Extending the Broad Histogram Method for Continuous Systems*
}

\author{
JOSE D. MUNOOZ ${ }^{1, \dagger}$ and HANS J. HERRMANN $\ddagger$ \\ Institute for Computer Applications 1, University of Stuttgart, \\ Pfaffenwaldring 27, D-70569 Stuttgart, Germany \\ ${ }^{1}$ Permanent address: Dpto. de Física, Univ. Nacional de Colombia, \\ Bogota D.C., Colombia \\ †E-mail: jdmunoz@ica1.uni-stuttgart.de \\ ${ }^{\ddagger}$ E-mail: hans@ica1.uni-stuttgart.de
}

October 17, 2018

\begin{abstract}
We propose a way of extending the Broad Histogram Monte Carlo method (BHMC) to systems with continuous degrees of freedom, and we apply these ideas to investigate the three-dimensional XY-model. Our method gives results in excellent agreement with Metropolis and Histogram Monte Carlo simulations and calculates for the whole temperature range $1.2<T<4.7$ using only 2 times more computer effort than the Histogram method for the range $2.1<T<2.2$. Our way of treatment is general, it can also be applied to other systems with continuous degrees of freedom.
\end{abstract}

Usually one wants to calculate average values $<Q>_{T}$ of a certain quantity $Q$ for a system in equilibrium at temperature $T$ according to, for instance, the canonical ensemble

$$
<Q>_{T}=\frac{\sum_{E}<Q>_{E} g(E) \exp \left(-E / k_{B} T\right)}{\sum_{E} g(E) \exp \left(-E / k_{B} T\right)},
$$

where $g(E)$ is the number of states with energy $E,<Q>_{E}$ denotes the micro-canonical average of $Q$ at energy $E$, and $k_{B}$ is the Boltzmann constant

\footnotetext{
* Oral presentation on CCP 1998, 2-5 September 1998, Granada, Spain
} 
(in the rest $k_{B}=1$ is taken). It is clear that given $g(E)$ and $\left\langle Q>_{E}\right.$ one can calculate $\left\langle Q>_{T}\right.$ at any desired temperature.

The Broad Histogram Monte Carlo Method (BHMC) developed by de Oliveira et. al. [1, 2, 3] calculates $g(E)$ and $\langle Q\rangle_{E}$ directly. It chooses a microreversible protocol of allowed virtual movements in the space of states of the system, and counts $N_{\text {up }}(X)\left(N_{\mathrm{dn}}(X)\right)$ as the number of allowed movements that increases (decreases) the energy of the configuration $X$ by a fixed amount $\Delta E_{\mathrm{fix}}$. These movements are virtual, in the sense that they are never performed. They are introduced only to estimate $g(E)$ and should not be mixed with the dynamics employed to take the samples. It has been shown [3] that the total number of possible ways to go up, summed over all states with energy $E$, equals the total number of ways to go down, summed over all states with energy $E+\Delta E_{\text {fix }}$, i.e.

$$
g(E)<N_{\text {up }}(E)>=g\left(E+\Delta E_{\text {fix }}\right)<N_{\text {dn }}\left(E+\Delta E_{\text {fix }}\right)>\quad,
$$

where $<N_{\text {up }}(E)>\left(<N_{\mathrm{dn}}(E)>\right)$ is the micro-canonical average of $N_{\text {up }}(X)$ $\left(N_{\mathrm{dn}}(X)\right)$ at energy $E$. By taking logarithms of both sides of Eq. (2) and by subsequent dividing by $\Delta E_{\mathrm{fix}}$ one can obtain expressions for $\ln g\left(E+\Delta E_{\mathrm{fix}}\right)-$ $\ln g(E)$ and $\beta(E) \equiv d \ln g(E) / d E$ that can be either added up or integrated numerically to obtain $\ln g(E)$. The micro-canonical averages involved in the method can be calculated either analytically or by Monte Carlo simulations. In this case four histograms are required, i.e. $N_{u p}(E), N_{d n}(E), Q(E)$ and the number of visits $V(E)$. This procedure gives accurate results with small computer efforts for many discrete systems [1, 3].

To extend the BHMC method to continuous systems we propose to choose a protocol of virtual random movements in the space of states of the system, such that for each allowed movement the probability to perform it equals the probability to revert it, i.e.

$$
P\left(X_{\text {old }} \rightarrow X_{\text {new }}\right)=P\left(X_{\text {new }} \rightarrow X_{\text {old }}\right) \quad .
$$

Next, let us define the probability density function (p.d.f.) $f^{X}(\Delta E)$ i.e. the probability to obtain an energy change between $\Delta E$ and $\Delta E+d \Delta E$ starting from the configuration $X$. We propose

$$
N_{\mathrm{up}}(X) \equiv f^{X}\left(\Delta E_{f i x}\right) \quad ; \quad N_{\mathrm{dn}}(X) \equiv f^{X}\left(-\Delta E_{f i x}\right) \quad .
$$

Eq. (2) is still valid, and the method proceeds as before [4]. 
To test our ideas we chose the XY-model [5] with the Hamiltonian

$$
\mathcal{H}=-J \sum_{<i j>} \vec{\sigma}_{i} \cdot \vec{\sigma}_{j}=-J \sum_{<i j>} \cos \left(\theta_{i}-\theta_{j}\right)
$$

where the summations $\langle i j\rangle$ are taken over all pairs of nearest-neighbor sites, $\theta_{i}$ denotes the planar angle of the spin at site $i$ and $J$ is the maximal energy per bond (in the rest $J=1$ ). As protocol of virtual random movements, we take at random one site $i$ with angle $\theta_{i \text { old }}$ and choose a new angle $\theta_{i \text { new }}$ for it with uniform probability on $[-\pi,+\pi]$. We obtain [4]

$$
f^{X}(\Delta E)=\frac{1}{N} \sum_{i} f^{i}(\Delta E) \quad ; \quad f^{i}(\Delta E)=\left\{\begin{array}{cc}
\frac{1}{\pi} \frac{1}{\sqrt{A_{i}^{2}-\left(\varepsilon_{i}+\Delta E\right)^{2}}} & \left|\Delta E+\varepsilon_{i}\right|<A_{i} \\
0 & \text { otherwise }
\end{array}\right.
$$

where $N$ denotes the number of sites $i$, and

$$
\varepsilon_{i} \equiv-\sum_{j} \cos \left(\theta_{i \mathrm{old}}-\theta_{j}\right) \quad ; \quad A_{i} \equiv \sqrt{\left(\sum_{j} \cos \theta_{j}\right)^{2}+\left(\sum_{j} \sin \theta_{j}\right)^{2}}
$$

The summation over $j$ is always performed on all nearest neighbors of site $i$. Finally $N_{\mathrm{up}}(X)\left(N_{\mathrm{dn}}(X)\right)$ equals the average of $f^{i}\left(\Delta E_{\mathrm{fix}}\right)\left(f^{i}\left(-\Delta E_{\mathrm{fix}}\right)\right)$ on all sites.

To estimate the micro-canonical averages we binned the whole energy range of positive temperatures (i.e. energies per bond between -1.0 and $0.0)$ in windows of size $\Delta E_{f i x}$ and performed a micro-canonical simulation on each window as follows [2]. Starting from a configuration inside the window one spin was rotated to a new angle chosen at random with uniform probability over the interval $[-\pi, \pi]$. If the new system energy falls inside the window, the change is performed, otherwise it is rejected. It is clear that this sampling process maintains a detailed balance condition with equal probabilities for the old an the new configurations and, therefore, it samples all the states inside the window with the same probability. An entire lattice sweep was performed by repeating this procedure on all sites.

Fig. 1 compares the specific heat we obtained for the SC $10 \times 10 \times 10$ $\mathrm{XY}$-model with periodic boundary conditions by using the BHMC method (circles), the Histogram Monte Carlo method (HMC) [7, 8] (squares) and Metropolis [6] simulations (diamonds). For the BHMC method $\Delta E_{f i x}=6.0$ $\left(\Delta E_{f i x}=0.002\right.$ in energy-per-bond units) was chosen.

The HMC method took 188 sec. per run, a Metropolis for one temperature point 28.5 sec. and the BHMC method 383 sec. per run on a Digital 

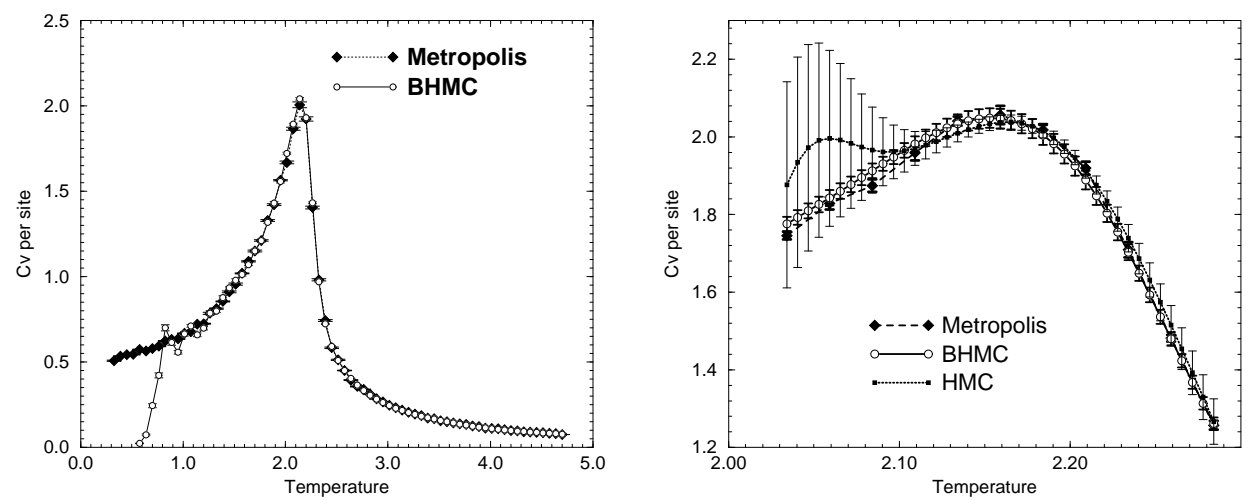

Figure 1: Specific heat for the SC $10 \times 10 \times 10 \mathrm{XY}$-model obtained from the BHMC method (circles), the HMC method (small squares) and Metropolis simulations (filled diamonds). The error bars on the left (right) side correspond to 1.0 (3.5, i.e. $99 \%$ confidence level) standard deviations for eight runs.

Alpha workstation, i.e. only 2.0 times more computer effort than the HMC method and less than 14 Metropolis simulations. It can be observed that all methods coincide on the temperature range $2.1<T<2.2$, i.e. the classical range of validity for this HMC simulation [10], but the BHMC method is still precise on the whole temperature range $1.2<T<4.7$.

In conclusion, our proposal to apply the BHMC method to continuous systems gives excellent agreement with Metropolis and HMC simulations. The strategy proposed is completely general, it can also be applied to other systems with continuous degrees of freedom.

\section{References}

[1] P.M.C. de Oliveira, T.J.P. Penna and H.J. Herrmann, Braz. J. of Phys. 26, 677-683 (1996); also in cond-mat/9610041.

[2] P.M.C. de Oliveira, Int. J. Mod. Phys. C9, 497 (1998).

[3] P.M.C. de Oliveira, to appear in Eur. Phys. J. B; also in condmat/9807354.

[4] J.D. Muñoz and H.J. Herrmann, to appear in Int. J. Mod. Phys. C; also in cond-mat/9810024.

[5] J. Adler et. al., Physica A 201, 581 (1993). 
[6] N. Metropolis et. al., J. Chem. Phys. 21, 1087 (1953).

[7] Z.W. Salsburg et. al., J. Chem. Phys. 30, 65 (1959).

[8] A.M. Ferrenberg and R.H. Swendsen, Phys. Rev. Lett. 61, 2635 (1988).

[9] W.H. Press et. al., Numerical Recipes in Fortran 90, 2nd. Ed. (Cambridge University Press, New York, 1996).

[10] M.E.J. Newman and R.G. Palmer, Error estimation in the histogram Monte Carlo method, Preprint cond-mat/9804306. 